\title{
Study of the performance of the ATLAS muon spectrometer
}

\author{
Cesare Bini
}

\author{
Sapienza Università and INFN, Roma \\ on behalf of the ATLAS Collaboration
}




\section{Detection of muons @ ATLAS}

Muons@LHC:

- probes of Standard Model processes: $Z \rightarrow \mu \mu, W \rightarrow \mu v, H \rightarrow \mu \mu \mu \mu$

- signature of phenomena beyond the Standard Model ( $Z^{\prime}, W^{\prime}$, SUSY)

How muons are detected:

- filtering provided by the calorimeters

- tracking in B field for momentum measurement

- matching with Inner Detector (ID) to improve resolution and vertex capabilities

The ATLAS muon spectrometer (MS): based on air-core toroid magnetic field:

- Detects muons up to $|\eta|=2.7\left({ }^{*}\right)$

- Triggers on muons (single and di-muon)

- Standalone operation + extrap. to vertex

- Combined mode with ID

(*) $\eta=-\log \tan \frac{\theta}{2}$
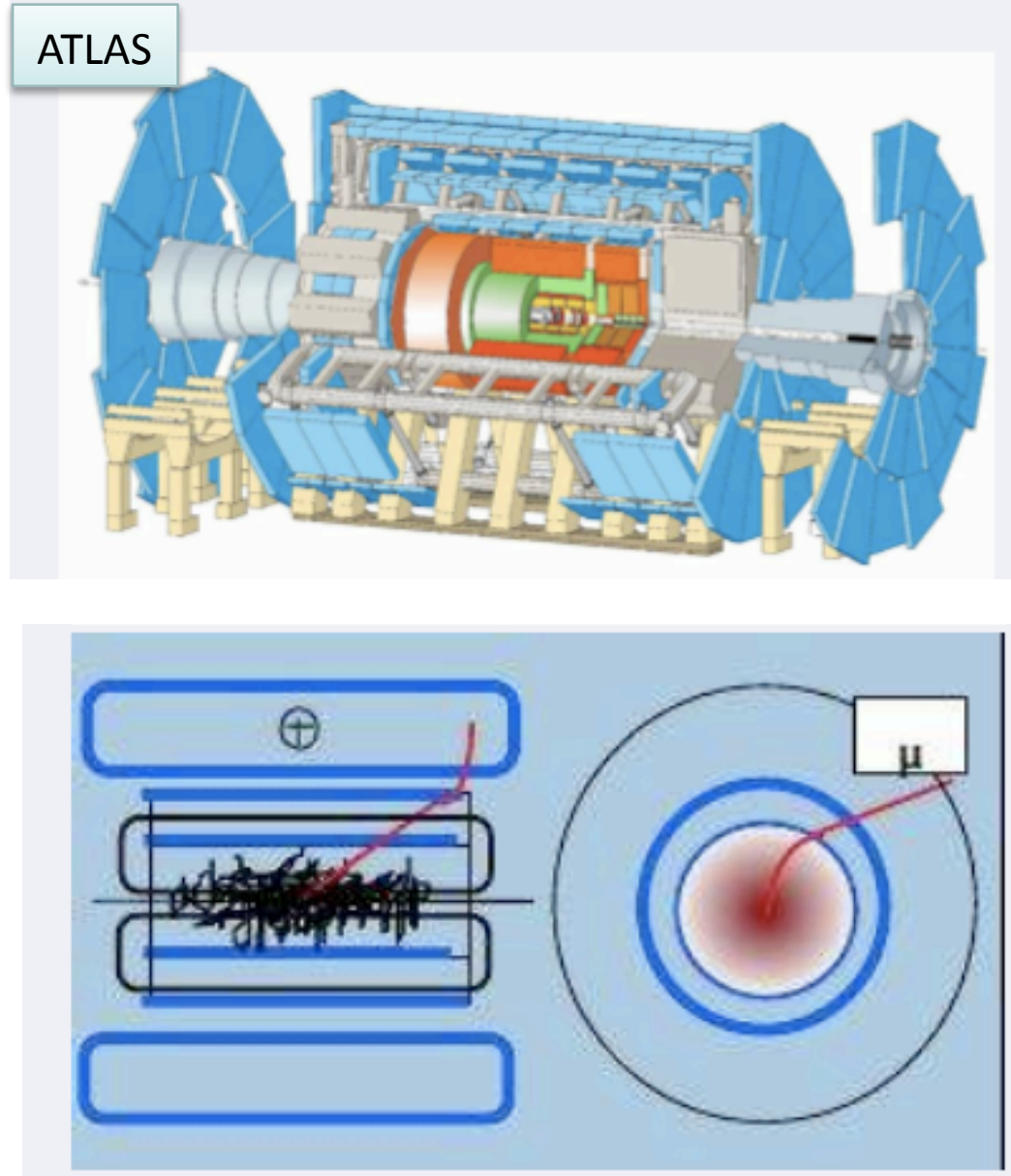


\section{$Z Z^{(*)} \rightarrow \mu^{+} \mu^{-} \mu^{+} \mu^{-}$event detected by ATLAS}

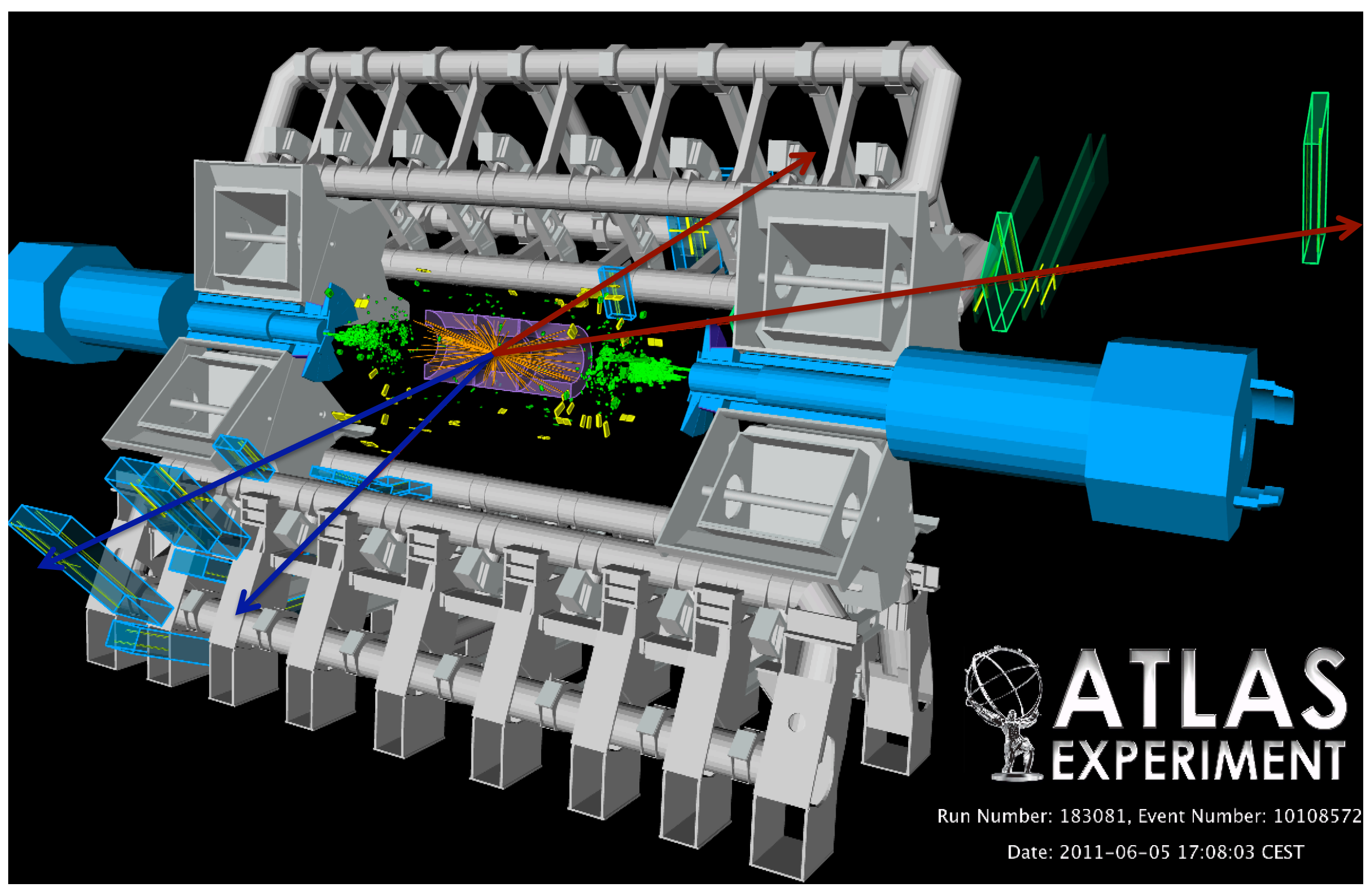




\section{The ATLAS Muon Spectrometer - I}

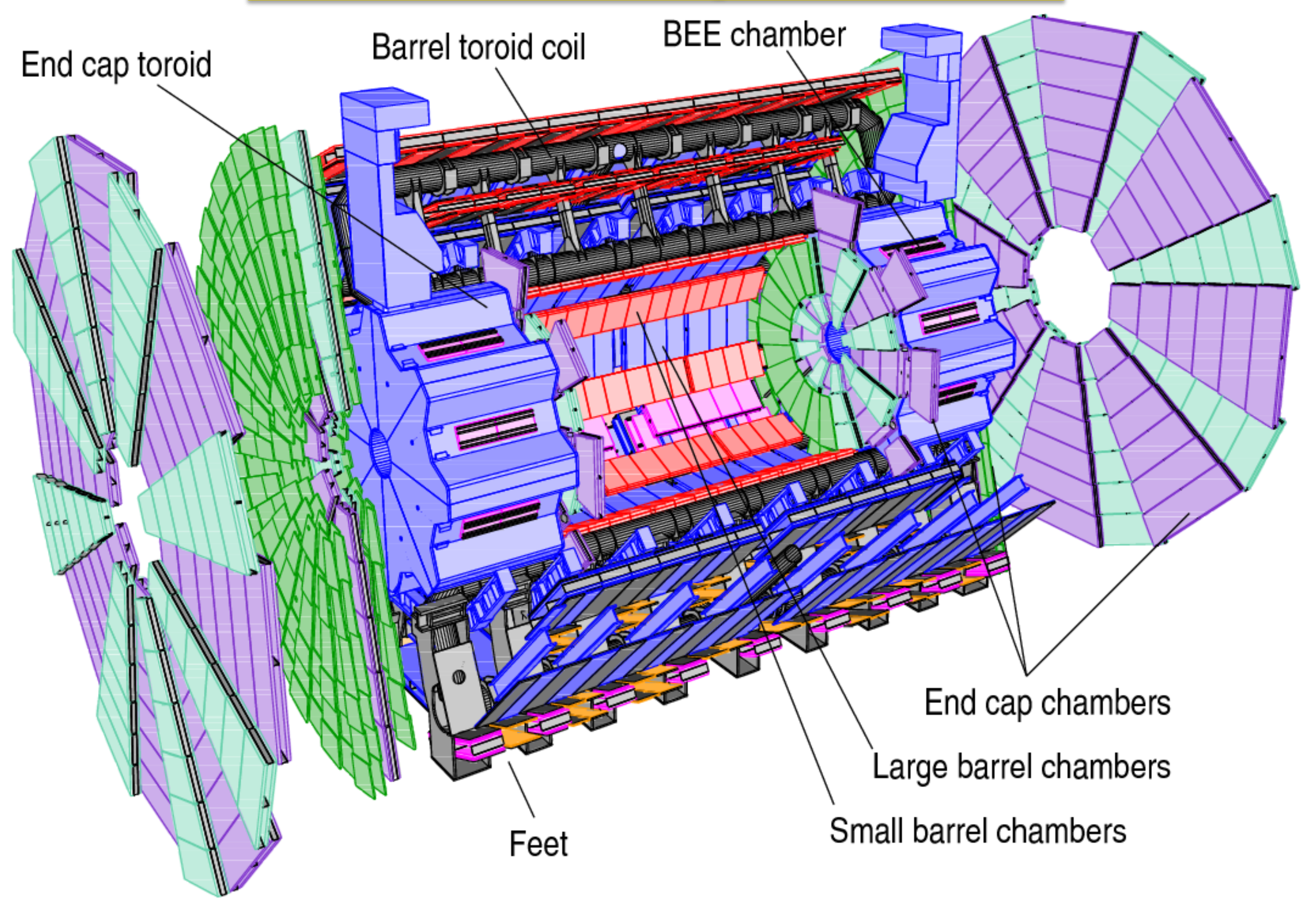




\section{The ATLAS Muon Spectrometer - II}
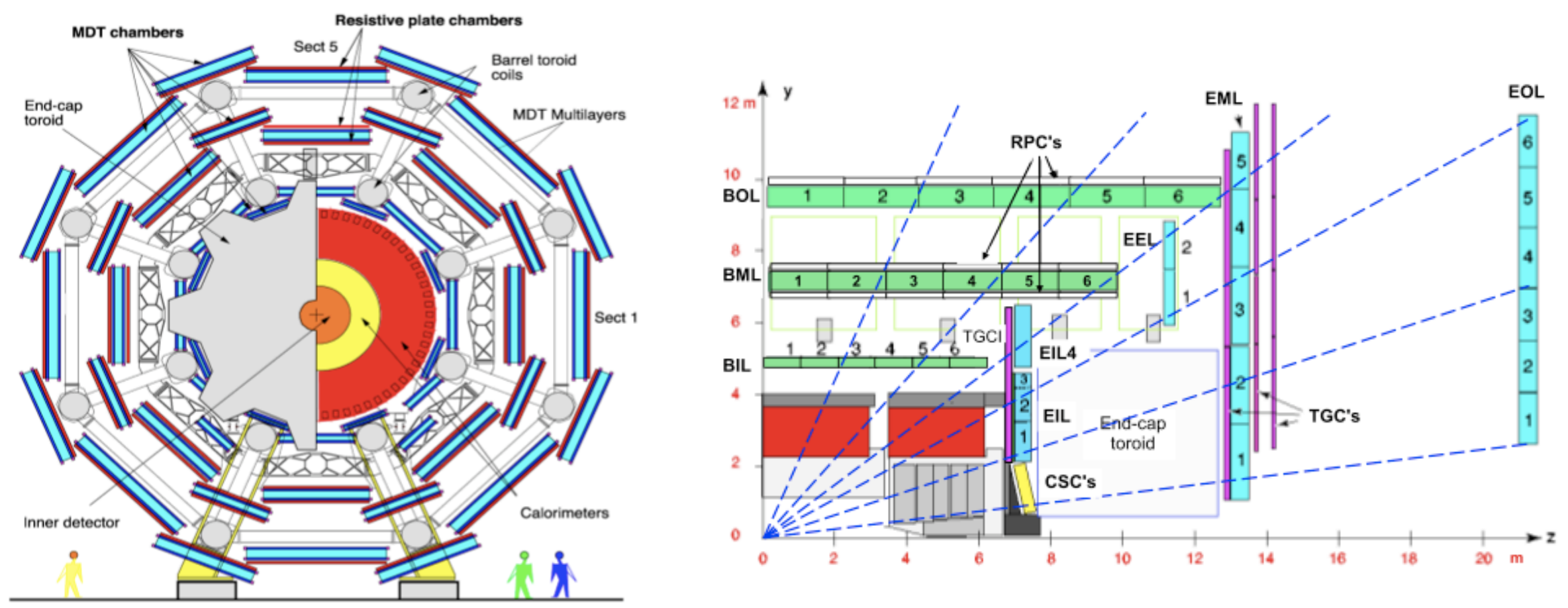

\begin{tabular}{|c|c|c|c|c|c|c|c|c|}
\cline { 2 - 8 } \multicolumn{2}{c|}{} & \multicolumn{2}{c|}{ Chamber resolution (RMS) in } & Measurements/track & \multicolumn{2}{c|}{ Number of } \\
\hline Type & Function & $z / R$ & $\phi$ & time & barrel & end-cap & chambers & channels \\
\hline MDT & tracking & $35 \mu \mathrm{m}(z)$ & - & - & 20 & 20 & $1088(1150)$ & $339 \mathrm{k}(354 \mathrm{k})$ \\
CSC & tracking & $40 \mu \mathrm{m}(R)$ & $5 \mathrm{~mm}$ & $7 \mathrm{~ns}$ & - & 4 & 32 & $30.7 \mathrm{k}$ \\
RPC & trigger & $10 \mathrm{~mm}(z)$ & $10 \mathrm{~mm}$ & $1.5 \mathrm{~ns}$ & 6 & - & $544(606)$ & $359 \mathrm{k}(373 \mathrm{k})$ \\
TGC & trigger & $2-6 \mathrm{~mm}(R)$ & $3-7 \mathrm{~mm}$ & $4 \mathrm{~ns}$ & - & 9 & 3588 & $318 \mathrm{k}$ \\
\hline
\end{tabular}




\section{ATLAS Muon Trigger concept}

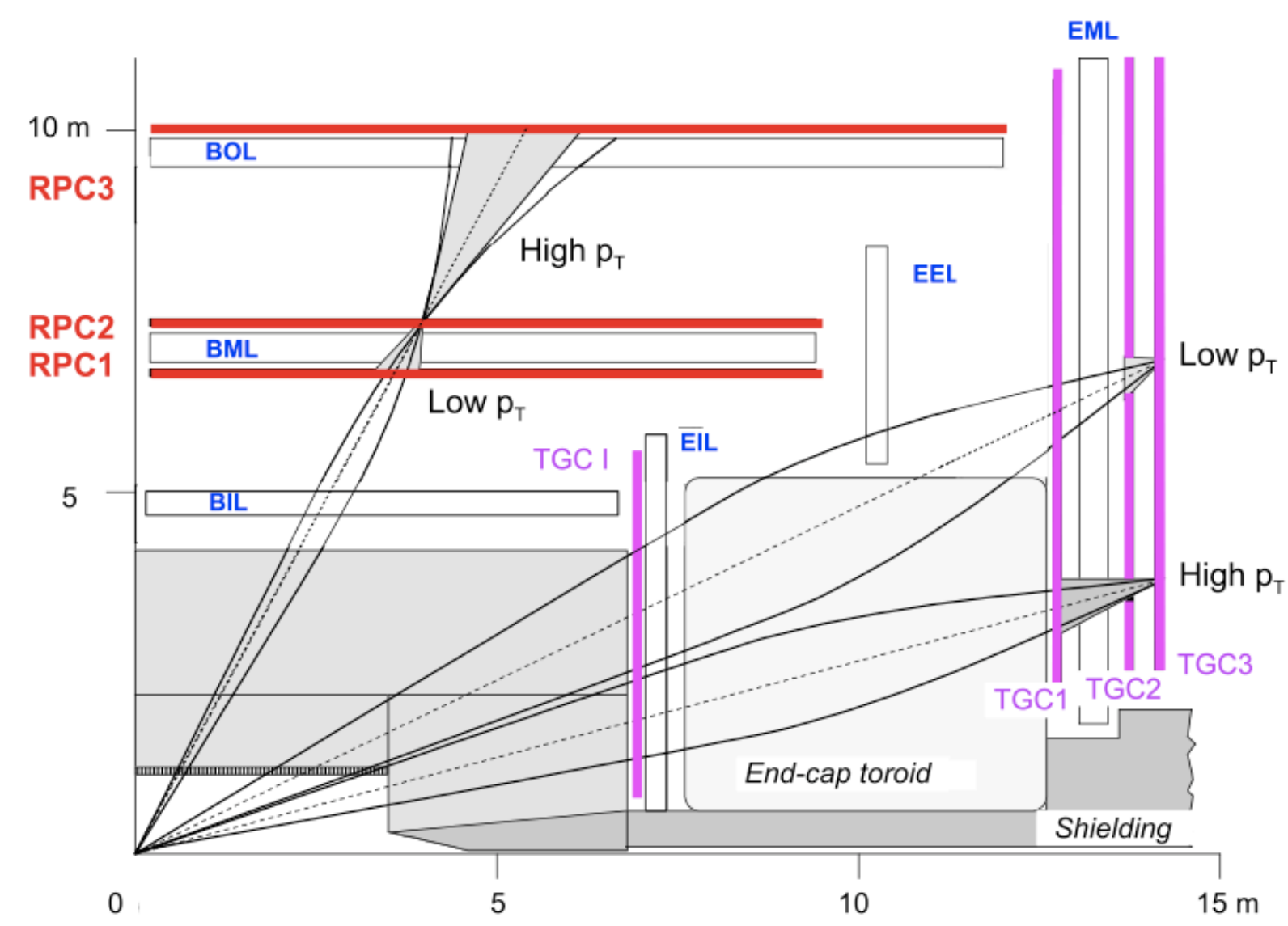

Level-1: hardware - based on RPC or TGC coincidences, with up to $6 \mathrm{p}_{\mathrm{T}}$ thresholds with full $\eta$ coverage;

Level-2: software - using precision chambers with coarse granularity Event-Filter: software - using quasi-offline track reconstruction

\begin{tabular}{|l|l|}
\hline & rate \\
\hline L1 & $<10 \mathrm{kHz}$ \\
\hline L2 & $<1 \mathrm{kHz}$ \\
\hline EF & $<100 \mathrm{~Hz}$ \\
\hline
\end{tabular}




\section{ATLAS Muon Tracking concept}

A muon tracks can be:

"standalone" based on MS

"combined" btw MS and ID

"tagged" ID + MS tag Tagged muons allow to recover "geometrical" inefficiencies The combined momentum resolution is dominated by

ID @ low $\mathrm{p}_{\mathrm{T}}$

MS@ high $\mathrm{p}_{\mathrm{T}}$

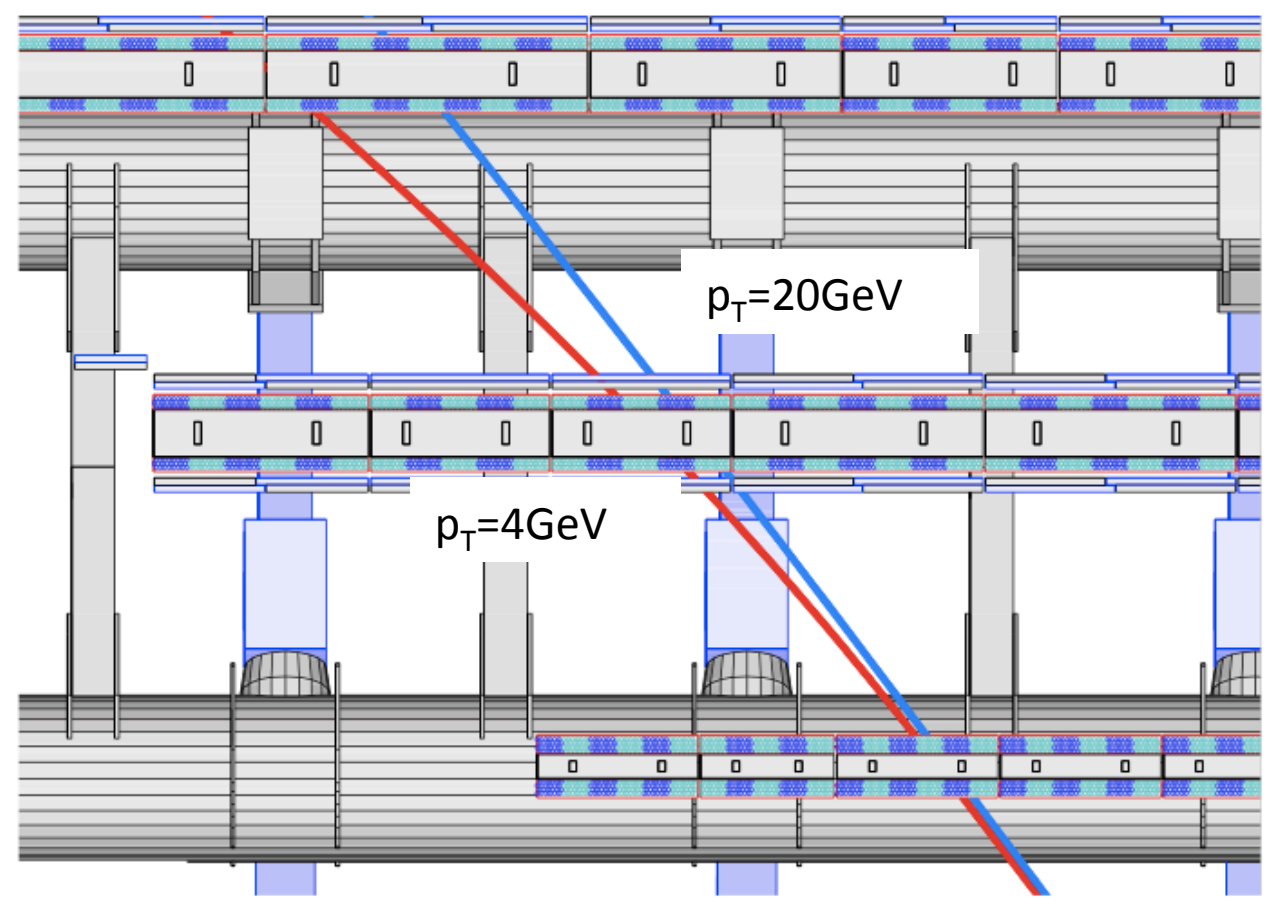

Design $\mathrm{p}_{\mathrm{T}}$ resolutions: standalone and combined (G.Aad et al., JINST 3,:S08003,2008)
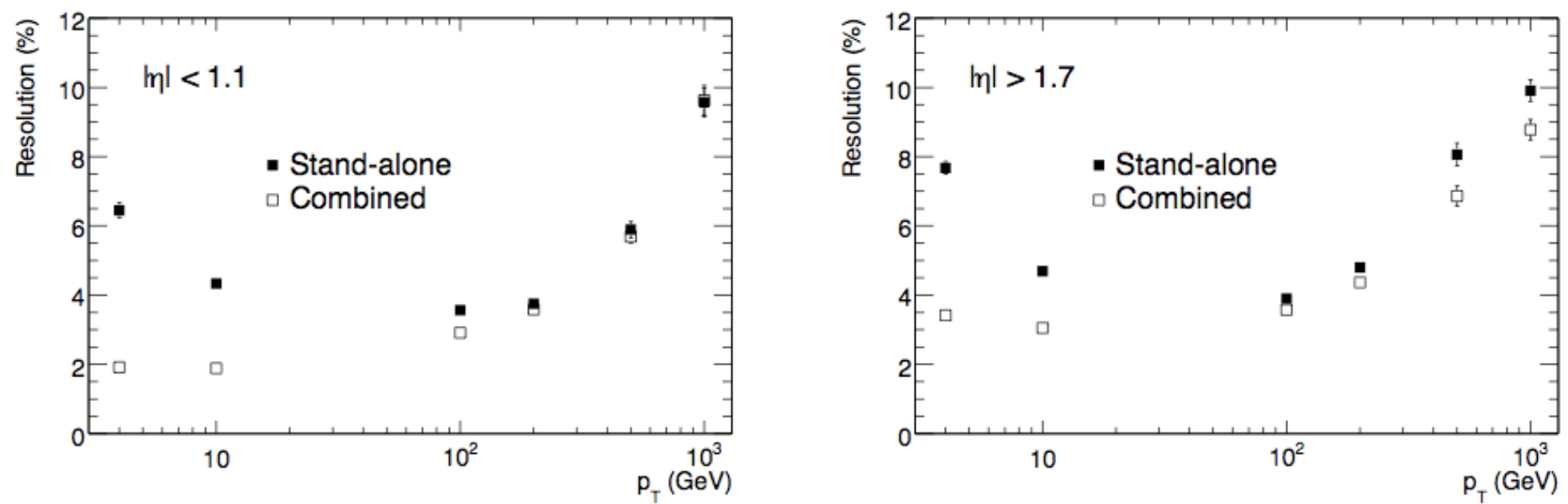


\section{Measurement of the muon performance}

The muon detection performance are continuously monitored during collision data-taking

$\rightarrow$ Data Quality Assessment and continuous Detector Calibration (see M.Iodice talk in this session)

$\rightarrow$ Tag \& Probe method to determine reconstruction and trigger efficiencies, based on $\mathrm{J} / \psi$ and $\mathrm{Z}$ resonances

$\rightarrow$ Resonance mass peaks and ID vs. MS comparisons to determine resolution and momentum scale

$\rightarrow$ Special runs with toroid off and solenoid on to determine alignment 


\section{Reconstruction Efficiencies: high $\mathrm{p}_{\mathrm{T}}$ muons}
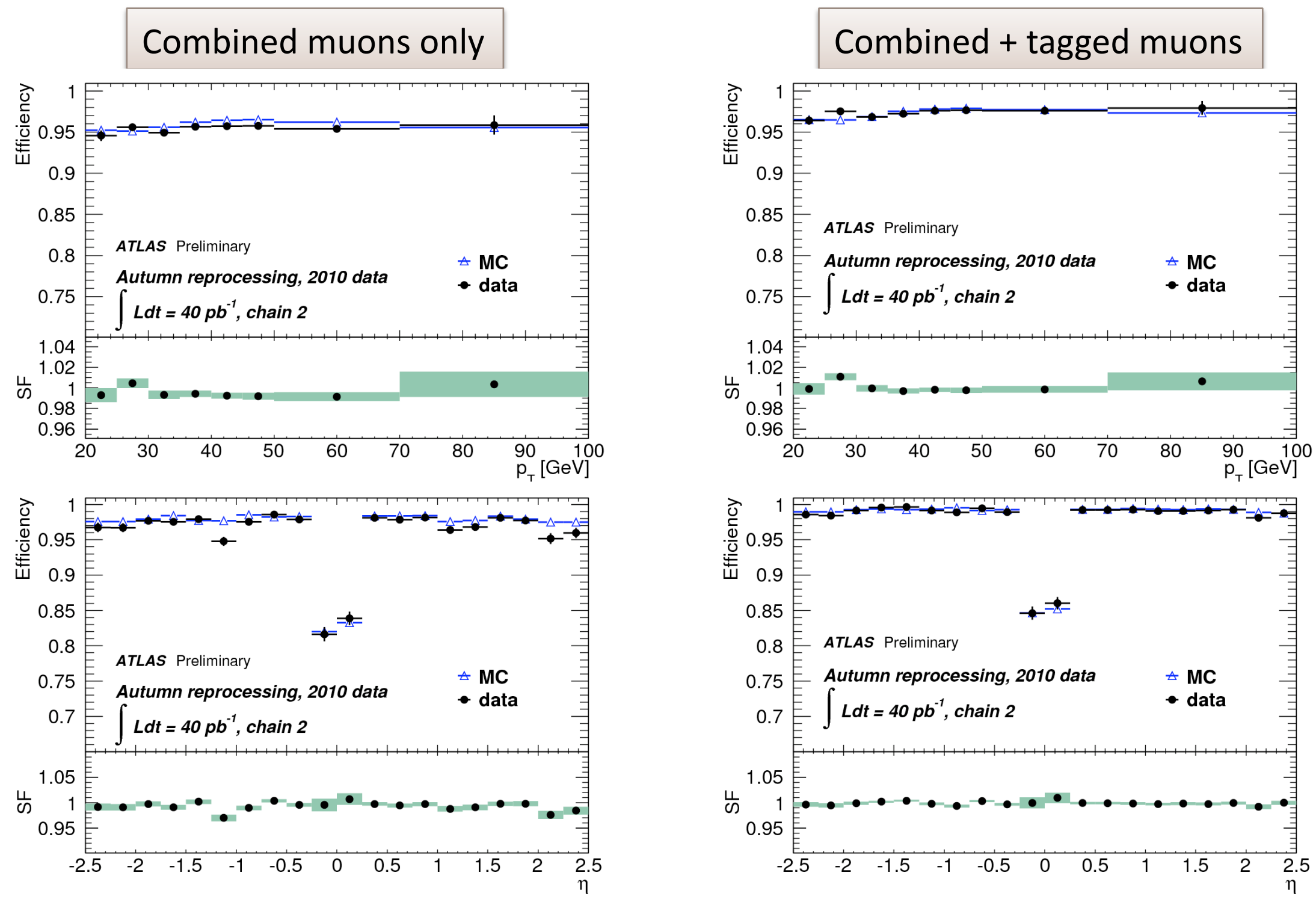

Measurements based on Tag\&Probe using the $\mathrm{Z}$ resonance $\rightarrow$ high efficiency and good data-MC agreeement. 


\section{Reconstruction Efficiencies: low $\mathrm{p}_{\mathrm{T}}$ muons}
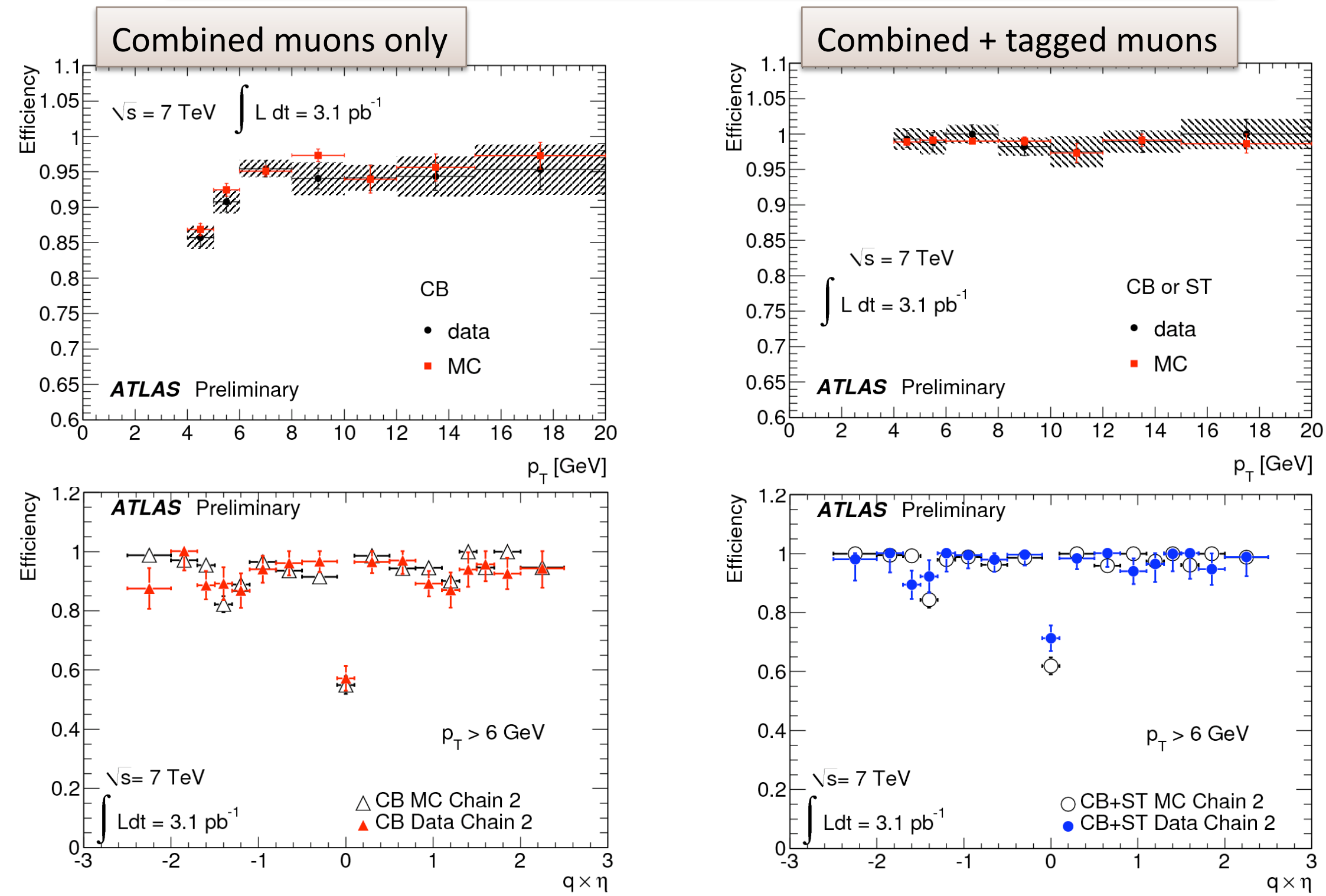

The measurements are based on Tag\&Probe using the $\mathrm{J} / \psi$ resonance $\rightarrow$ high efficiency for $\mathrm{p}_{\mathrm{T}}>6 \mathrm{GeV}$ and good data-MC agreeement. 


\section{Trigger Efficiencies}

Trigger efficiencies are evaluated using the Tag\&Probe method on the $\mathrm{Z}$ with respect to combined tracks $\rightarrow \varepsilon$ ( Trigger / reconstruction )

Results of the measurement for the single muon trigger with threshold $=18 \mathrm{GeV}$
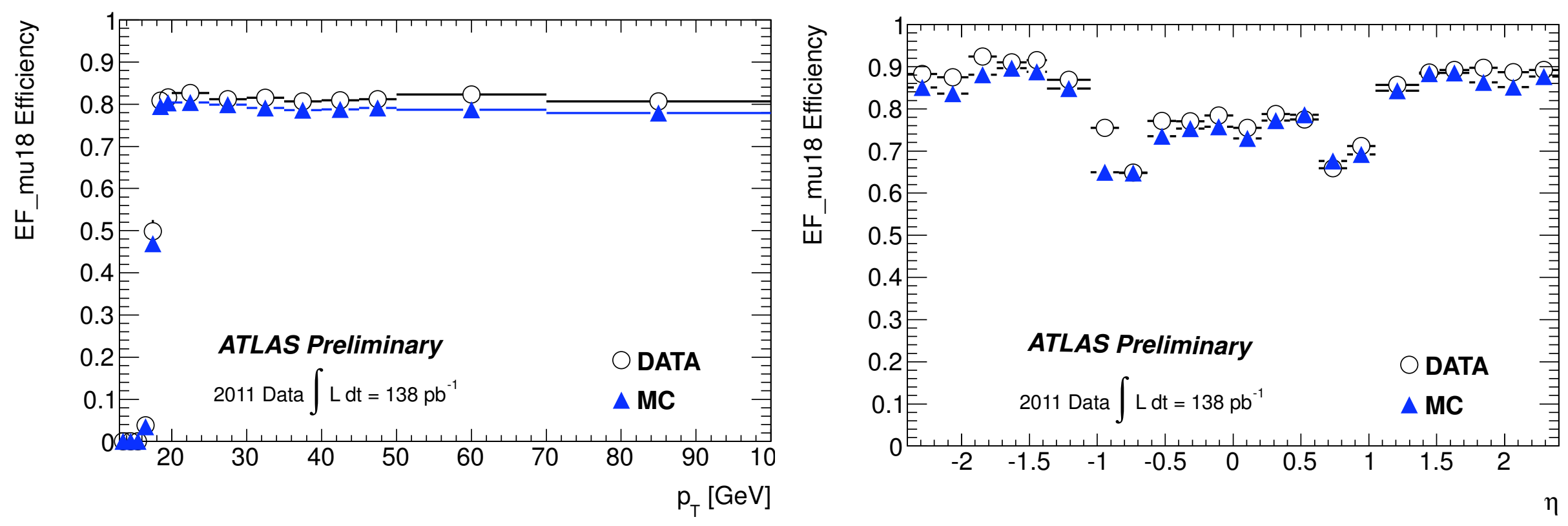

The $80 \%$ efficiency at the plateau is due to regions with limited acceptance (clearly identified in the $\eta$ distribution) 


\section{Muon Spectrometer Resolution: 2010 data}

MS momentum resolution as a function of $\mathrm{p}_{\mathrm{T}}$ for Barrel and Endcap constrained by $Z \rightarrow \mu \mu$ line-shape and by ID vs. MS measurement from $W \rightarrow \mu \nu$ events.

$$
\frac{\sigma\left(p_{T}\right)}{p_{T}}=\frac{p_{0}^{M S}}{p_{T}} \oplus p_{1}^{M S} \oplus p_{2}^{M S} p_{T}
$$

$\boldsymbol{p}_{0}{ }^{M S} \rightarrow$ energy loss

$p_{1}^{M S} \rightarrow$ multiple scattering

$p_{2}{ }^{M S} \rightarrow$ hit resolution (calib. + alignm.)
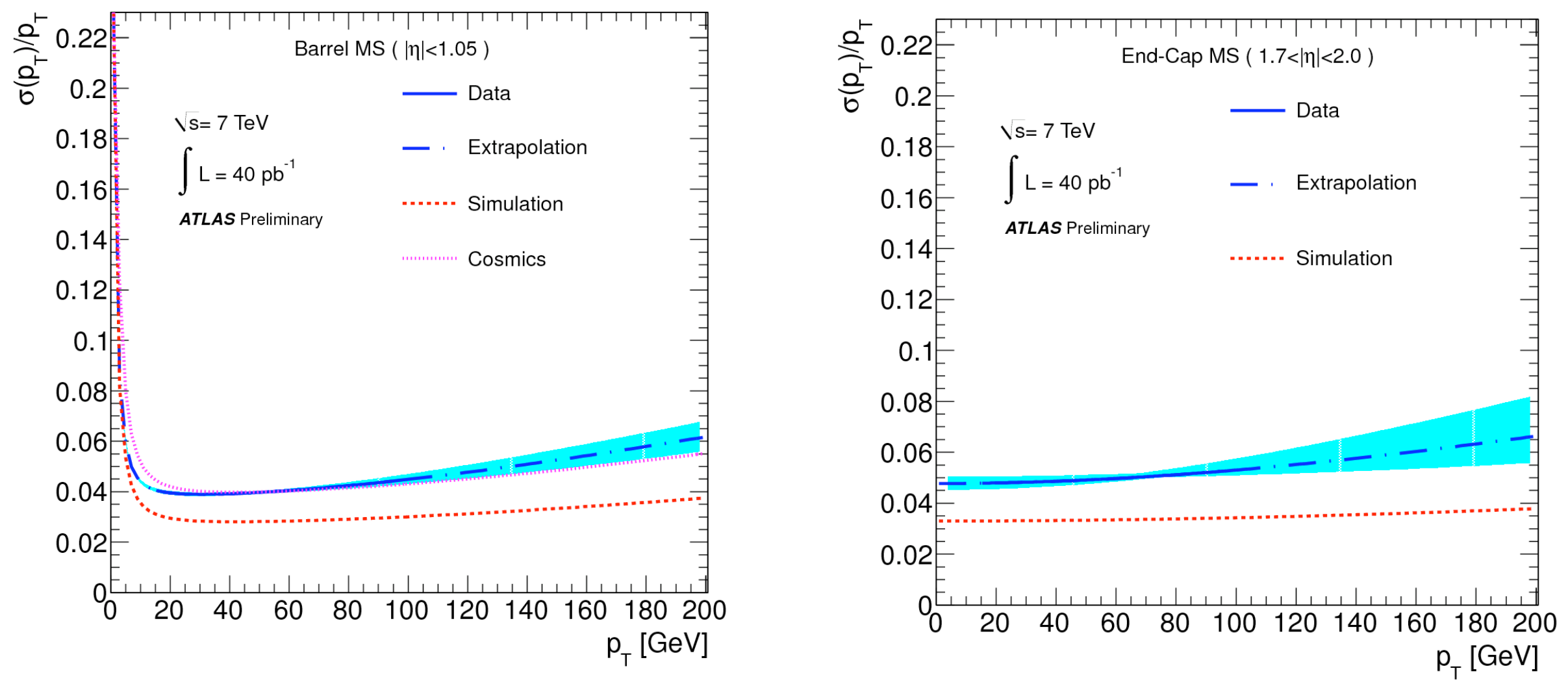

Improvement expected with new software release based on new alignments 


\section{Chamber alignment from toroid off data}

Alignment is based on:

(1) Optical sensors on all chambers

(2) Intercalibration using straight tracks (toroid off data) with high momentum The goal is $<50 \mu \mathrm{m}$ all over the detector (very challenging goal)

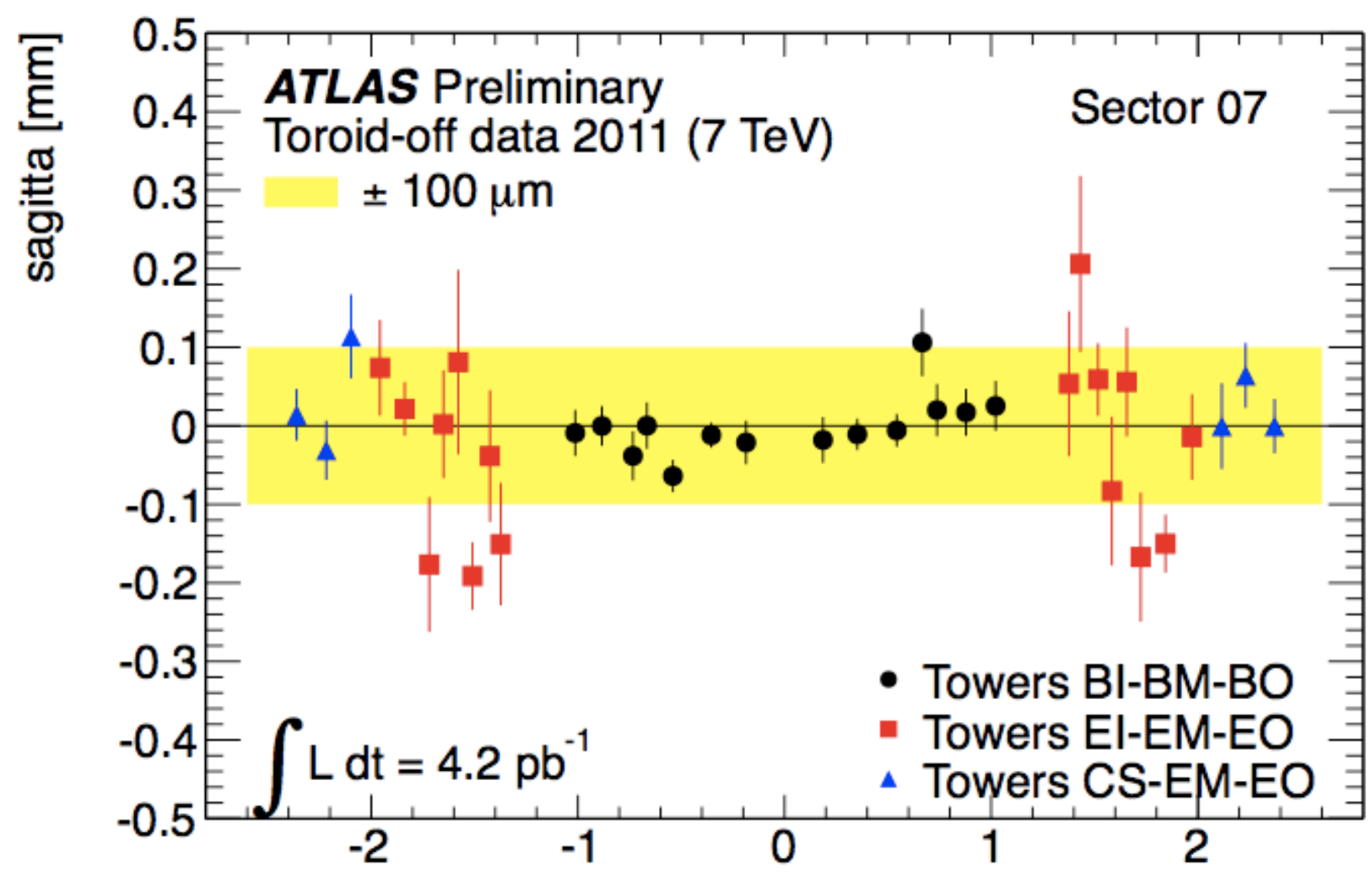

$\eta$

2011 data show an alignment within $50 \mu \mathrm{m}$ (barrel) and $100 \mu \mathrm{m}$ (endcap)

New alignment runs are expected to reduce the spread on endcap to $50 \mu \mathrm{m}$. 


\section{Conclusions and Outlook}

Many physics results from ATLAS are based on muon detection:

$\rightarrow$ Trigger and Reconstruction efficiencies match well the design performance;

$\rightarrow$ Momentum resolution of ID and MS approaching design values;

$\rightarrow$ Work in progress to define the optimal alignment all over the detector.

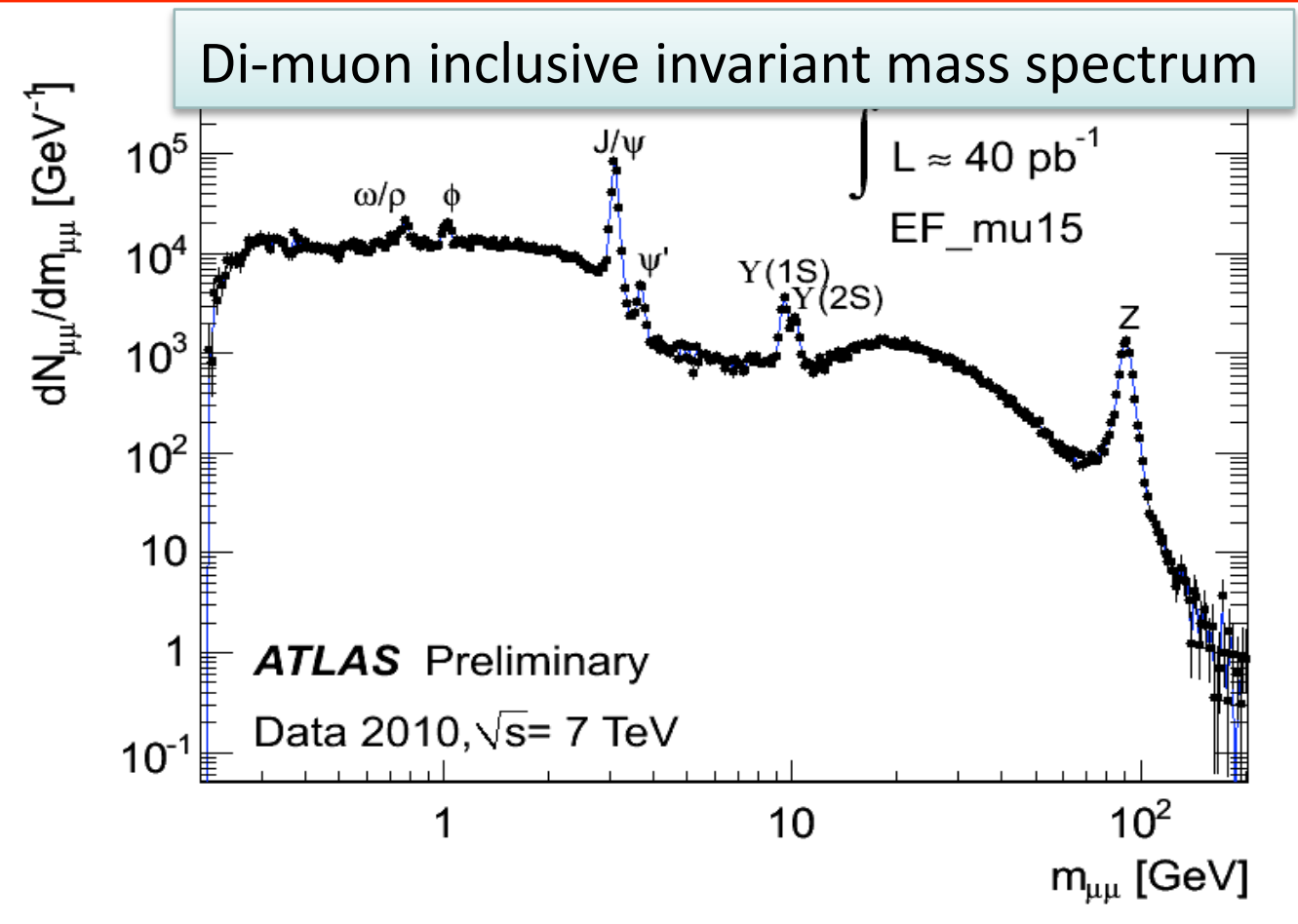




\section{Backup}




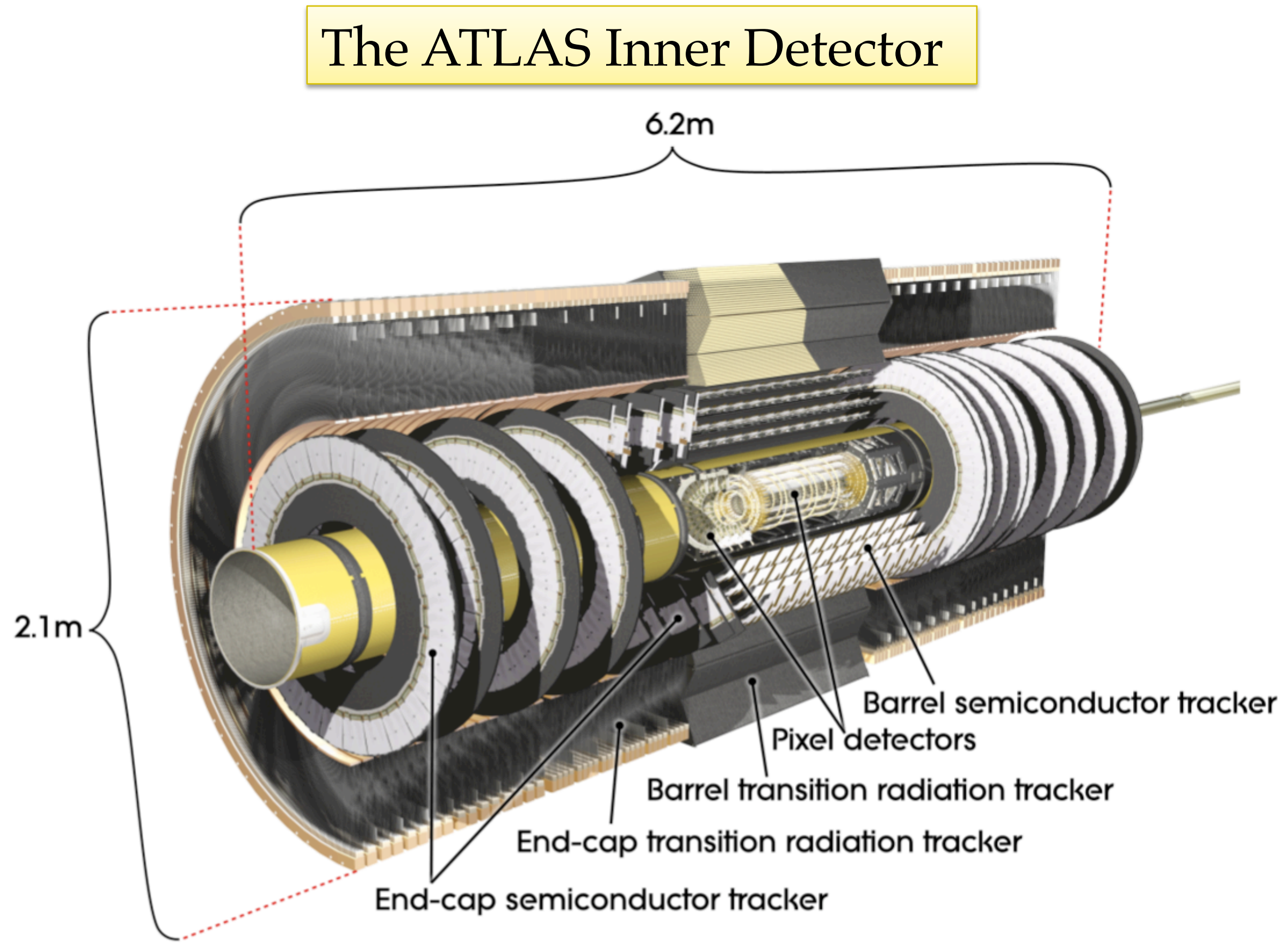




\section{Dimuon invariant mass spectrum}

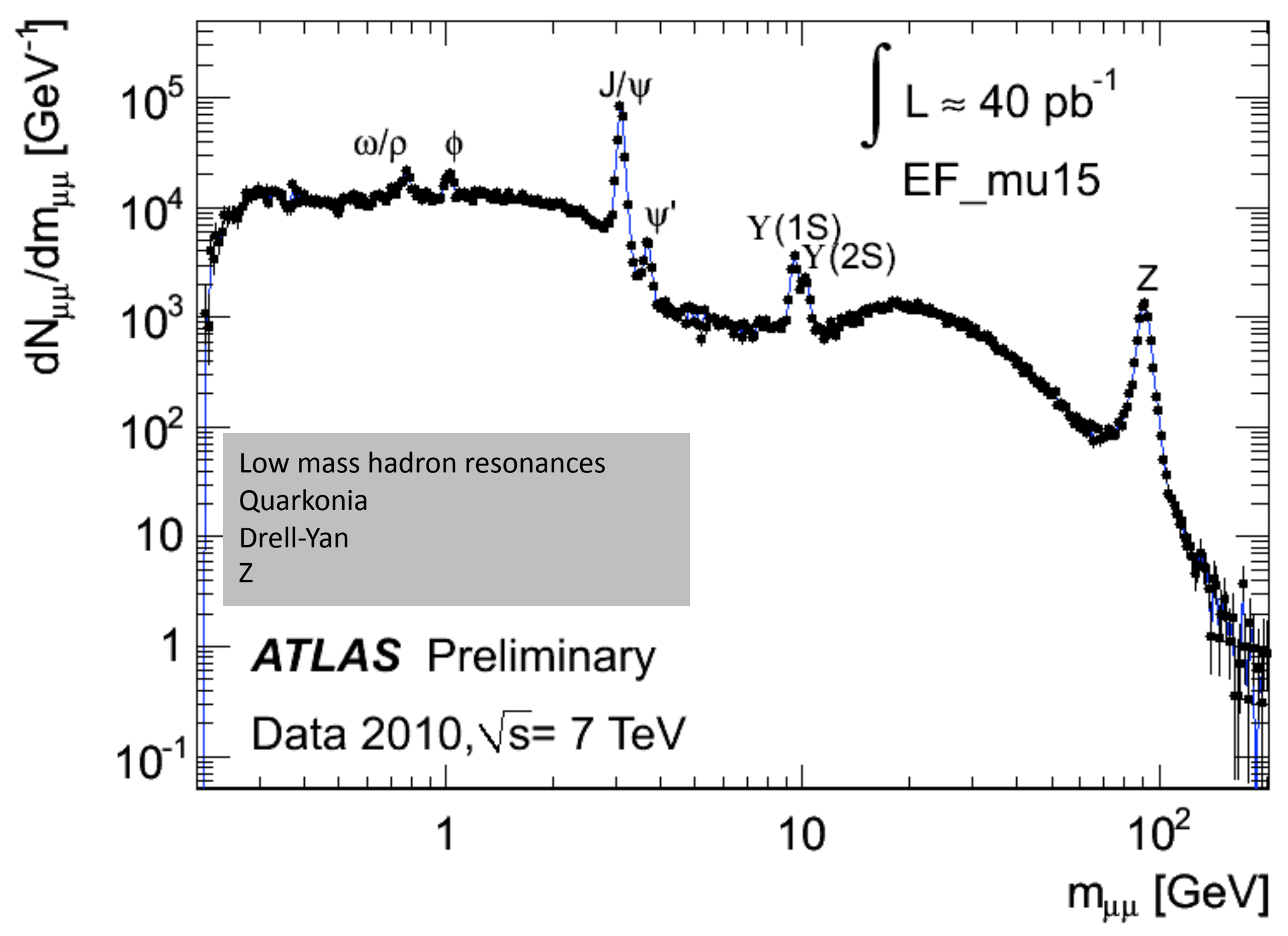




\section{ATLAS muon spectrometer technologies}

\section{Precision chambers}

(1) Monitored Drift Tubes (MDT)

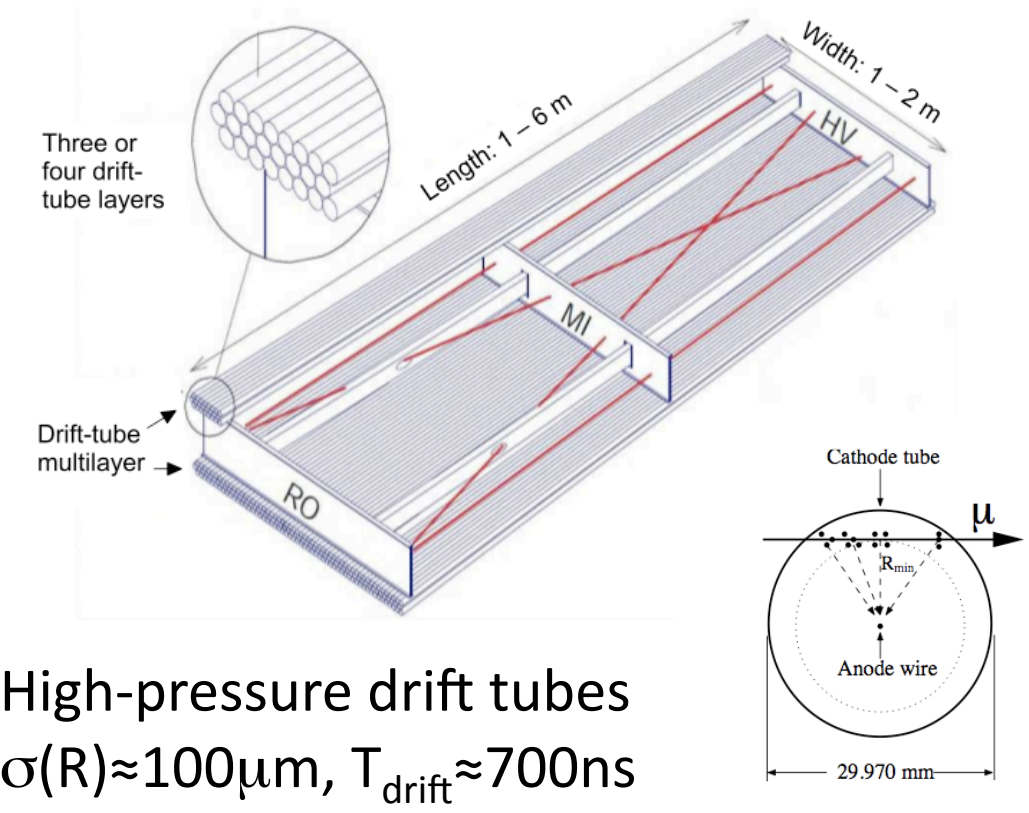

(2) Cathode Strip Chambers (CSC)

Operation in high rate environment $\sigma(\mathrm{R}) \approx 60 \mu \mathrm{mT} \mathrm{drift}_{\text {}} \approx 20 \mathrm{~ns}$

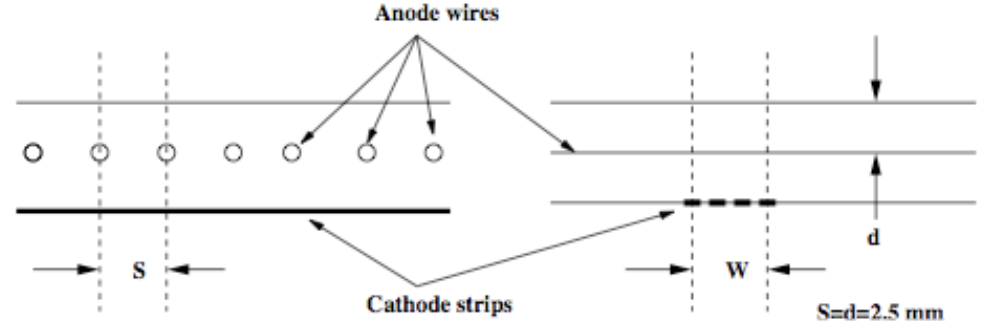

\section{Trigger chambers}

(1) Resistive Plate Chambers (RPC)

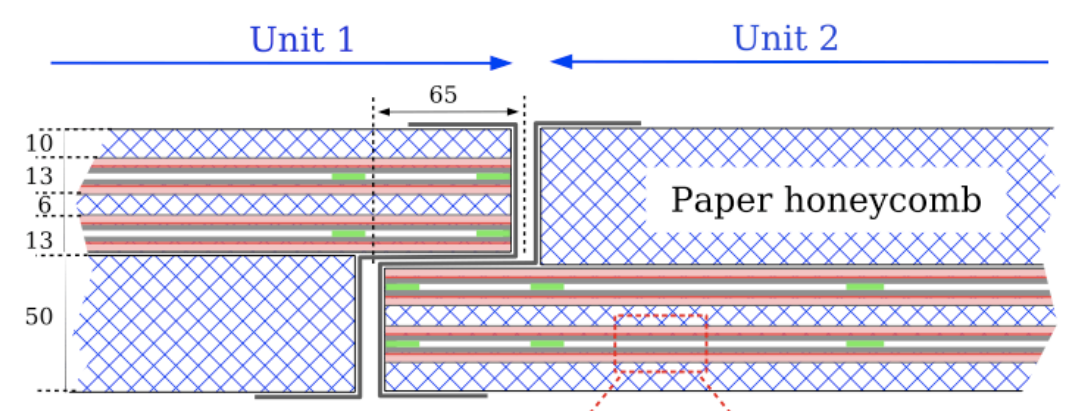

Schematic, not to scale

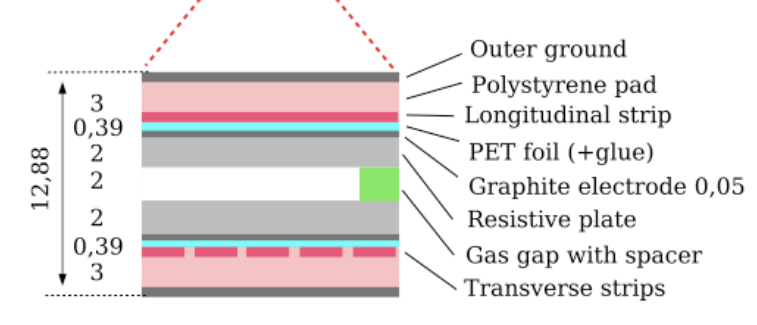

(2) Thin Gap Chambers

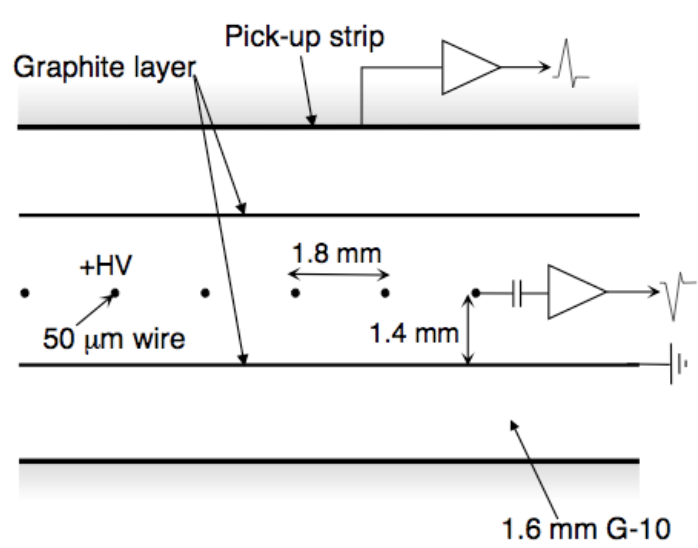




\section{$\mathrm{Z}$ mass resolution after alignment (2010 data)}
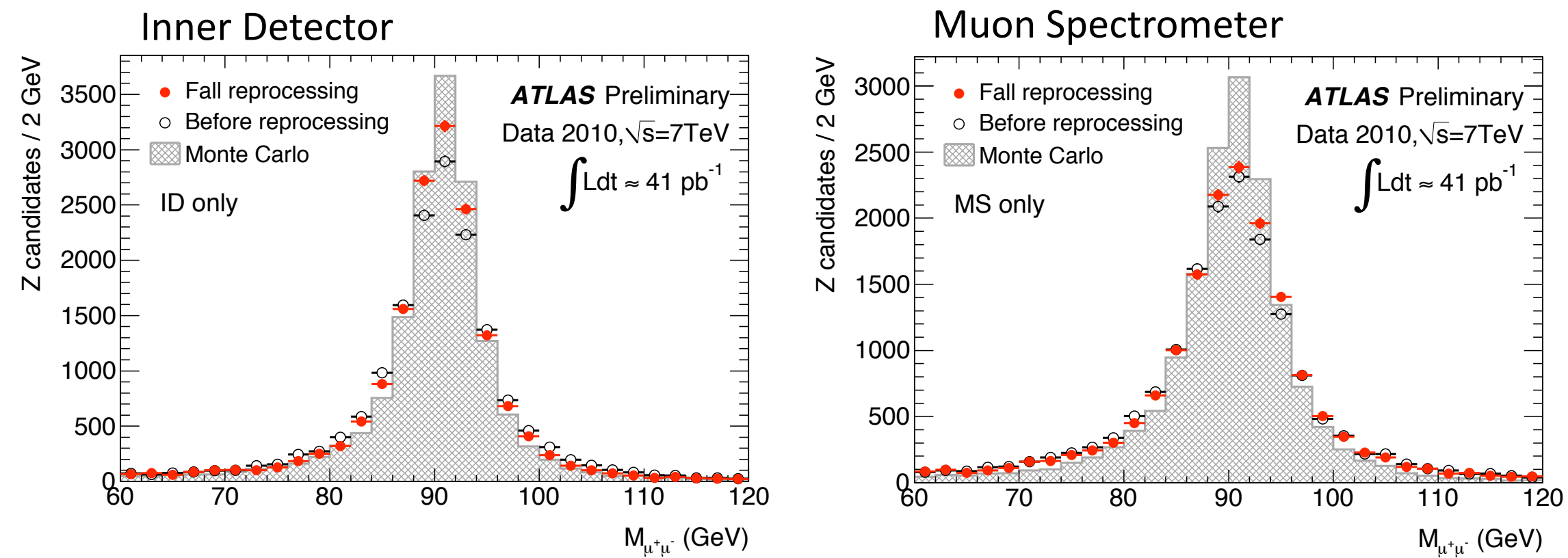


\section{$Z$ invariant mass resolution: comparison with $\mathrm{MC}$ results based on perfect alignment}
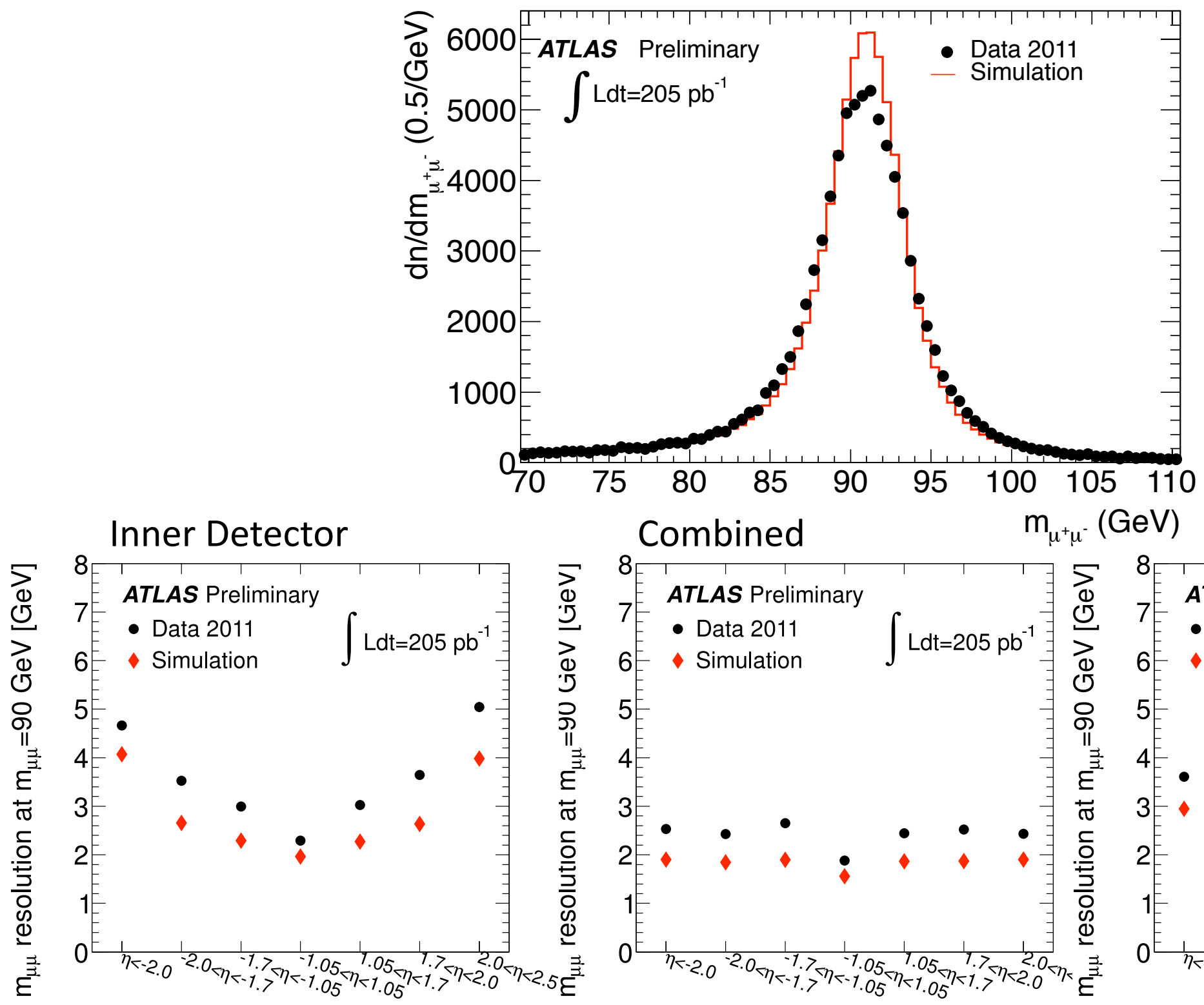

(GeV) Muon Spectrometer 


\section{Muon Spectrometer Resolution: 2010 data}
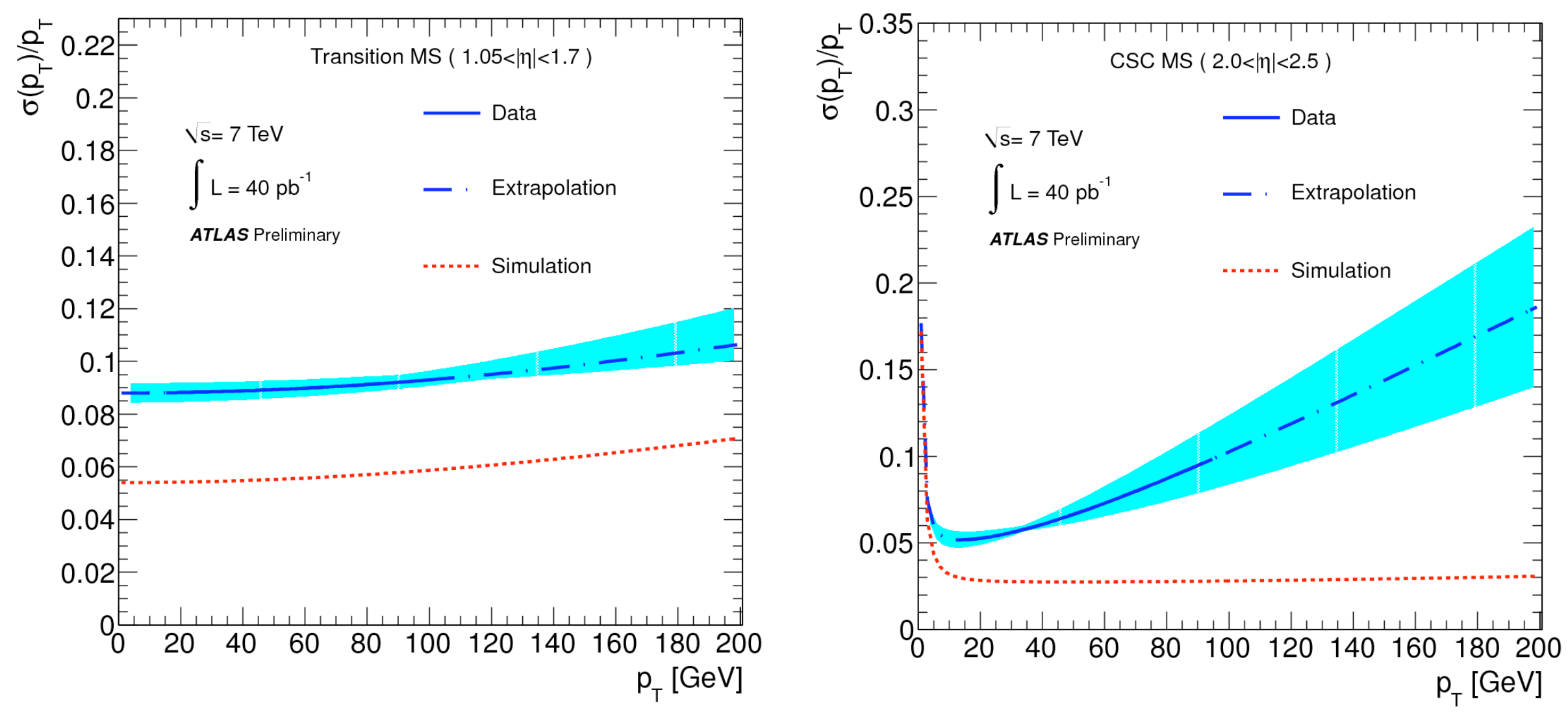


\section{Inner Detector Resolution: 2010 data}
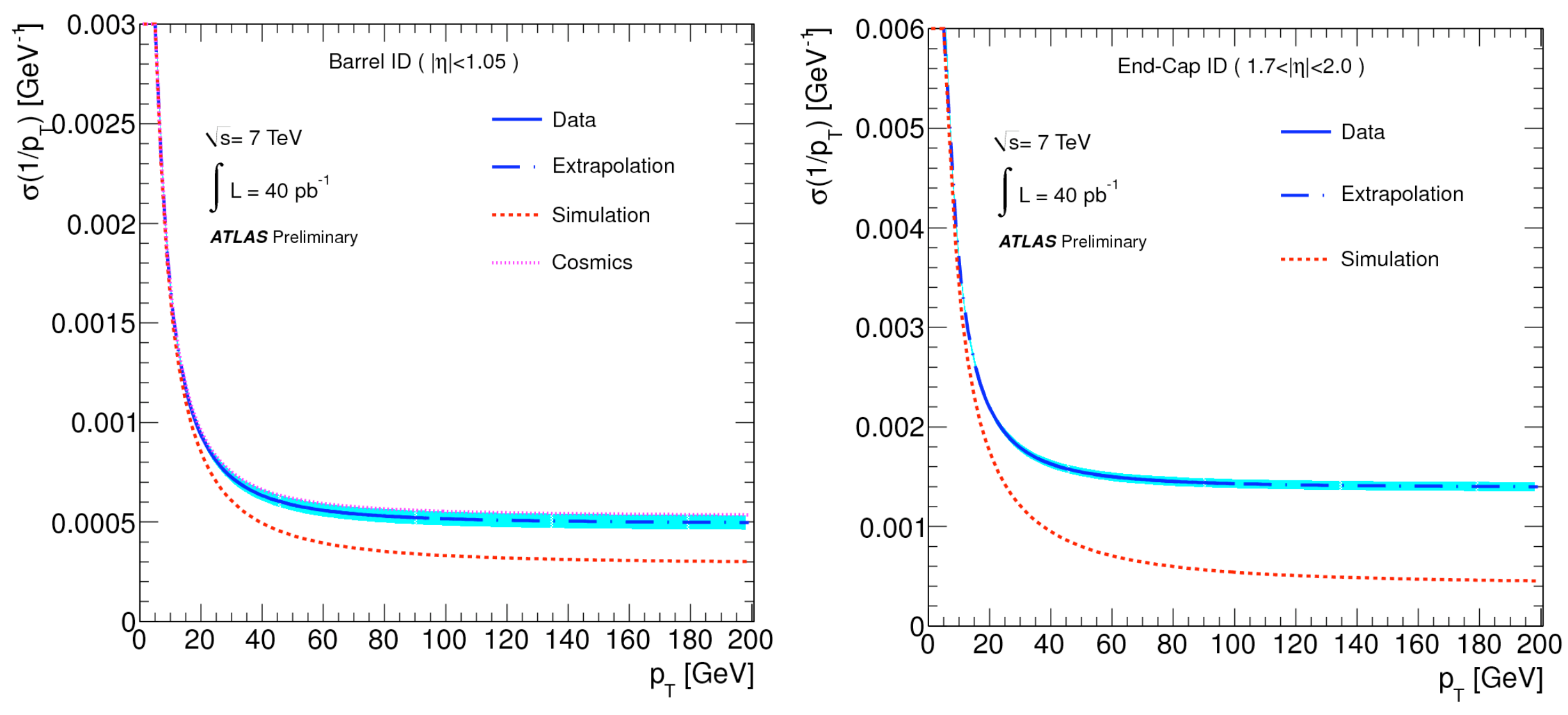
Sagitta measurements of straight tracks in toroid off data: Comparison between use of nominal geometry and aligned geometry.
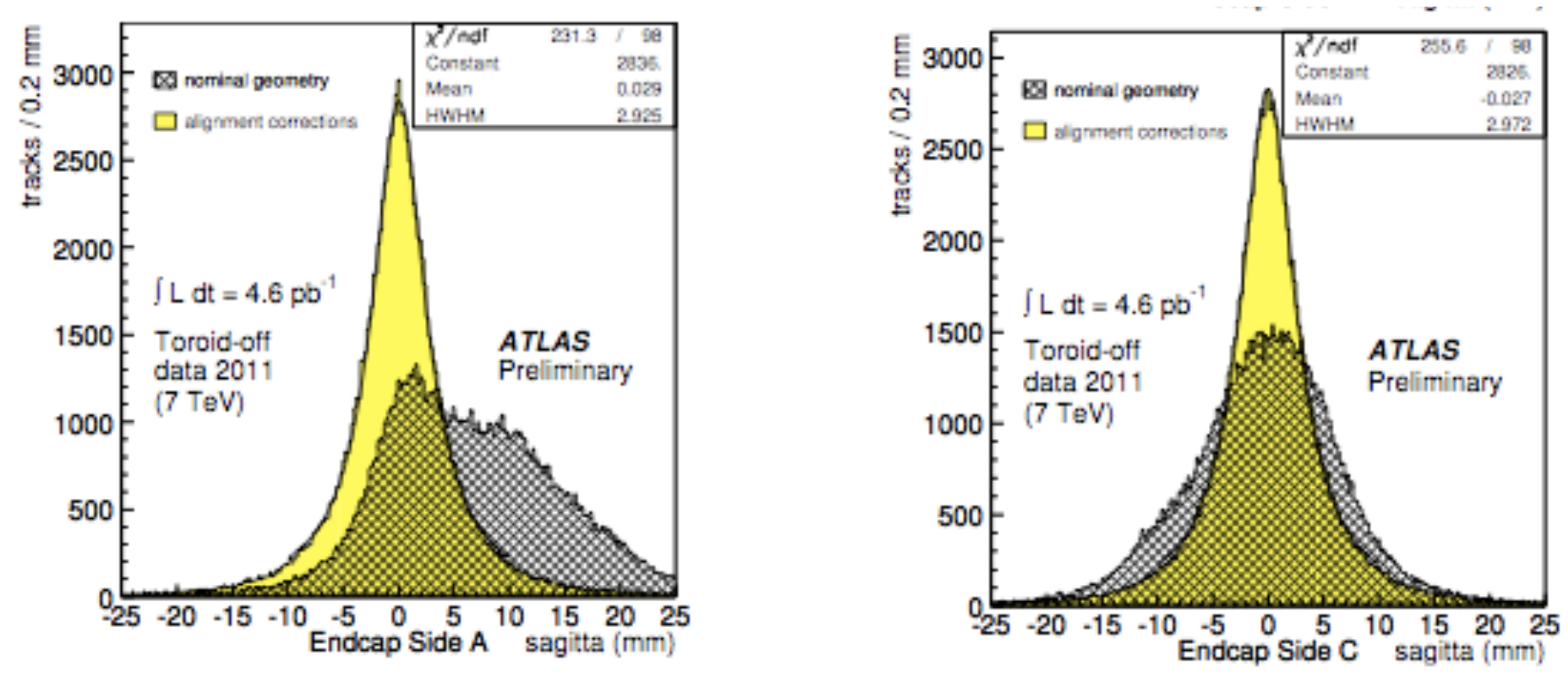